\title{
EP-28
}

\section{Analysis of lesions of liver in autopsy}

\author{
Amar RANJAN ${ }^{* 1}$, Harshita DUBEY', Pranay TANWAR', Abhishek YADAV ${ }^{2}$
}

${ }^{1}$ Department of Laboratory Oncology, Dr. BRA Institute-Rotary Cancer Hospital, All India Institute of Medical Sciences, New Delhi, India

${ }^{2}$ Department of Forensic Medicine, AlIMS, New Delhi, India

Introduction: Lesions of liver cause significant morbidity and mortality. Liver biopsy may not give the exact picture on lesions of liver. Autopsy study may be a better choice for this. The aims and objectives are: 1) to analyze lesions of liver in autopsy in India; 2) to establish the usefulness of autopsy pathology in clinical practice, teaching medical students and for research purposes.

Methods: Retrospective analysis of histopathological findings of autopsy cases was done for the year 2017 to 2020. Inclusion criteria were cases showing positive histopathological finding in any of the following organs heart, lung, liver, spleen, kidney, and brain. Total 70 cases were taken out. The age ranged from 4 days to 77 years. Male and female were 52 and 18 respectively. Out of total 70 cases, 40 showed lesions in liver.

Results: Most common lesion was Hepatic steatosis with foci of cirrhosis 14/70 (20\%). This was followed by cirrhosis (9/70)12.8\%, chronic hepatitis \& granuloma 4/70 (5.7\%) each, Cardiac cirrhosis (2/70), Nonalcoholic hepatic steatosis in an 11 months old male baby with umbilical granuloma, hydatid cyst, metastasis from primary cancer \& chronic hepatitis in 1/70 cases. Rest cases didn't show any pathology.

Conclusions: The pattern of liver diseases is different in the different ethnic groups. In India, no such study is available. Autopsy study may have clearer picture of lesions of liver than what we see in liver biopsy, which may be used for academic $\&$ research purposes. 